\title{
Usefulness of Cost Effectiveness: Evidence versus Applicability Godofreda Vergeire-Dalmacion*
}

Department of Pharmacology and Toxicology, College of Medicine, University of the Philippines Manila, Manila, Philippines

All human beings are created equal in terms of right to health and thus qualified to receive the best pharmaceutical option whether for prevention or cure. However, not all humans enjoy the same economic capability to procure their health care. To ensure that health care is inclusive, a responsible government actively assumes the task of procuring the drugs or biological for the health of its population while being mindful of ensuring rational spending and equitable distribution of resources. Cost effectiveness Analysis (CEA) has revolutionized the way doctors in the individual level and policymakers in the level of society should choose the best pharmaceutical intervention by estimating the cost per number of lives saved or cost per disability adjusted life years.

Given that drug safety is already established, benefits from treatment used to be the primal concern for decision making related to prescribing. Now, with uncontrolled escalation of drug prices and limited resources, cost of drugs or vaccines has become a direct determinant pushing for Cost Effectiveness Analysis (CEA). Had prices been the same among different vaccines, cost effectiveness studies would have been superfluous. Considering the differences in pharmaceutical prices and differences in expected clinical outcomes, the decision making process proceeds by comparing the extra benefit a patient gets from the extra cost of buying a more expensive treatment or preventive option [1]. But is cost effectiveness always reliable?

To answer this question, I dissected the elements in calculating effectiveness without necessarily doing the Math. To illustrate, I have taken the liberty of using the case of PCV10 versus PCV13 for immunization of children less than 5 years old in the Philippines to prevent pneumonia. However, the issues raised herein may already be immaterial since more body of evidence supports the use of PCV13. Nonetheless, these issues will be recurrent for CEA. Firstly, although the method for appraising evidence of effectiveness is objective, the choice and translation of the evidence is not. Evidence also changes through time based on new discovery or new studies and results of different studies looking at the same research question can contradict each other [2]. Allegedly, one of the reasons that a Secretary of Health in our country was summarily dismissed from Office was his controversial decision to procure Pneumonia Conjugate Vaccine (PCV) 10 over PCV13. Existing CEA studies showed that both PCV 10 and PCV13 were cost effective based on GDP per capita with PCV 13 moderately more cost- effective [3-6]. During this time, information about the protective effect of Protein D carrier unique to PCV10 against $H$. influenzae disease has yet to be explored. The gap in this knowledge maybe critical to countries like the Philippines with high burden of otitis media from $H$. influenzae but not to other countries with a different epidemiologic profile. Secondly, numbers are hypnotic and this can be unintentionally used as leverage. For example, the price difference between PCV 10 (15.40) from PCV 13 (16.34) was only 0.94 cents. When the cost of treating an episode of pneumonia, meningitis and sepsis is added, the cost of PCV10 increases dramatically to 1.6 billion USD versus the 1.3 billion for PCV13. The question is where the cost of illness was obtained? Thirdly, assumptions are aplenty in estimating cost effectiveness. In this case, it assumes that number of people in both arms who will actually seek treatment, will be hospitalized or lose productivity can be estimated. In making assumptions, tendency to overinflate the efficacy of the new and more expensive treatment is likely to be committed. Since therapeutic equipoise is the ethical and rational reason for discovering new treatment options, one can expect the result of any efficacy trial on the new drug to be almost always much better. Fourth, the more serious pitfall of appraising evidence of effectiveness is missing the details. In the case of the CEA presented to the Philippines Formulary Executive Council, the importance of the data on acute otitis media was marginalized. The World Health Organization (WHO) has identified the Philippines to be a high burden country for Chronic Suppurative Otitis Media (CSOM) which is a sequela from untreated or partially treated Acute Otitis Media (AOM). PCV 10 was shown to have averted 11 million cases of $\mathrm{AOM}$ versus to 7.3 million from PCV13. About $4 \%$ of AOM is expected to become CSOM which can lead to meningitis, deafness and brain abscess. Allegedly, $50 \%$ of cases of CSOM lead to sensory neural deafness. WHO revealed that CSOM in children is likely to inhibit language and cognitive development due to hearing loss. During the first two years of life both AOM and CSOM have been linked to persistent and significant hearing loss with learning disabilities and poor scholastic performance.

If the denominator for the CER (Cost effectiveness ratio) was "deaths averted "PCV13will appear better. But if the effectiveness was measured by "Disability adjusted life years averted" I surmise that the result will tilt in favour of PCV10. This was not evident in the report. Fifth the policymakers cannot be faulted for using intuition or the most uncommon common sense. Although the leading cause of deaths in under - five children are preterm birth complications, pneumonia, birth asphyxia, diarrhoea and malaria, about $45 \%$ of all child deaths are linked to malnutrition not infections. If money was not spent on health promotion for proper nutrition, the CEA becomes a mathematical exercise distant from the reason distant it was developed. But Pharmaceutical companies cannot sell nutrition. Lastly, coverage and willingness to pay can be equally important as CER for some decision makers. About $6 \%$ of the targeted children will not be immunized if PCV13 is purchased over PCV10. The trade-off may not be acceptable to policy makers who wish to expand coverage of health care delivery. In instances where both drugs and vaccines is cost effective, coverage may take priority over CER. Since CEA is not about money or finances, pay or can choose the least expensive option but with a lower incremental cost effectiveness ratio. Barring corruptions and the suspicion of bribery, CEA can guide policy makers toward a

*Corresponding author: Godofreda V Dalmacion, Department of Pharmacology and Toxicology College of Medicine, University of the Philippines Manila, Manila, Philippines, Tel: +639178408556; Fax: +6325254095; E-mail: jody.dalmacion@ gmail.com

Received November 26, 2015; Accepted January 05, 2016; Published January 09, 2016

Citation: Vergeire-Dalmacion G (2016) Usefulness of Cost Effectiveness: Evidence versus Applicability. Pharm Anal Acta 7: 456. doi:10.4172/21532435.1000456

Copyright: (c) 2016 Vergeire-Dalmacion G. This is an open-access article distributed under the terms of the Creative Commons Attribution License, which permits unrestricted use, distribution, and reproduction in any medium, provided the original author and source are credited. 
rational and objective process of decision- making.

I have not discussed cost because this is another contentious matter. It is almost unimaginable to compute for cost if the country does not have data on cost of illnesses or does not consistently record diagnosis using the International Classification of Diseases (ICD) Coding.

This commentary is not meant to discourage readers from conducting or using CEA for decision-making. Note that no actual calculation was included. Rather, it is meant to remind health professionals about the principles underpinning the CEA and its limitations. For the author, CEA is the first step but not the sole basis to deciding which the better therapeutic option is. The devil in the CEA is in the details which should be tackled by the Health Professionals. It should not be left alone to the Health Economists or Statisticians. Regardless of the weaknesses of CEA, it is a good springboard for decision making. On the individual level of prescribing, it is beneficial but from the societal level other objective factors must be considered and this is the task of the decision makers. Doctors should not only keep abreast with the latest, robust, relevant and reliable body of evidence and strive to possess the skill of translating knowledge. Doctors must keep in mind that health economics is a branch of social science and decision making using economic data always favours welfare of society.

\section{References}

1. Dhaliwal I, Duflo E, Glennester R, Tulloch C (2012) Comparative CostEffectiveness Analysis to Inform Policy in Developing Countries: A general Framework with Applications for education.

2. Weintraub WS, Cohen DJ (2009) The Limits of Cost-effectiveness analysis. Circ Cardiovasc Qual Outcomes 2: 55-58.

3. Ayieko P, Griffiths UK, Ndiritu M, Moisi J, Mugoya IK (2013) Assessment of Health-Benefits and Cost-Effectiveness of 10-Valent and 13- Valent Pneumococcal Conjugate Vaccination in Kenyan Children. PLoS One 8.

4. Boonacker CW, Broos PH, Sanders EA, Schilder AG, Rovers MM (2011) Costeffectiveness of pneumococcal conjugate vaccination against otitis media in children: a review. Pharmacoeconomics 29: 199-211.

5. (2012) Cost-effectiveness of Pneumococcal Conjugated Vaccination in the Philippines. Analysis of the World Health Organization and UP Manila.

6. Farkouh R, Hall-Murray C, Klok R, Hilton B, Isturiz R (2015) Comment on: Cost effectiveness Evaluation of the 10-Valent Pneumococcal NonTypeable Hemophilus Influenza Protein D Conjugate vaccine and 13 Valent Pneumococcal Vaccine in Japanese Children. Infect Dis Ther 4227-233. 